\title{
Properties of canonical fermion determinants with a fixed quark number
}

\author{
Julia Danzer* \\ Institute of Physics, University of Graz, Austria \\ E-mail: julia.danzer@uni-graz.at

\section{Christof Gattringer} \\ Institute of Physics, University of Graz, Austria \\ E-mail: christof.gatttringer@uni-graz.at
}

\section{Ludovit Liptak}

Institute of Physics, Slovak Academy of Sciences, Dubravska cesta 9, 84511 Bratislava, Slovakia and Institute of Physics, University of Graz, Austria

E-mail: Ludovit.Liptak@savba.sk

\begin{abstract}
Using a dimensional reduction formula for the lattice fermion determinant we study canonical determinants on quenched SU(3) gauge configurations. The canonical determinants decribe a fixed quark number and we analyze their properties below and above the transition temperature. We find that above $T_{c}$ the signatures of center symmetry breaking are very strongly manifest in the distribution of the canonical determinants in the complex plane, and we discuss possible physical implications of this finding. We furthermore analyze the relative weight of the different quark sectors below and above the transition temperature.
\end{abstract}

The XXVII International Symposium on Lattice Field Theory - LAT2009

July 26-31 2009

Peking University, Beijing, China

\footnotetext{
${ }^{*}$ This work is supported by FWF DK W 1203 .

† Speaker.

$\ddagger$ C.G. gratefully acknowledges support by the Dr. Heinrich Jörg Foundation.
} 


\section{Introductory comments about canonical determinants}

Canonical fermion determinants $\operatorname{det}[D]^{(q)}$, which describe a fixed number $q$ of quarks, are conceptionally interesting objects. On the lattice they may be obtained from the usual grand canonical determinant $\operatorname{det}[D(\mu)]$ as a Fourier transform with respect to imaginary chemical potential $\mu$ :

$$
\operatorname{det}[D]^{(q)}=\frac{1}{2 \pi} \int_{-\pi}^{\pi} d \varphi e^{-i q \varphi} \operatorname{det}[D(\mu=i \varphi / \beta)] .
$$

Here $\beta$ is the inverse temperature which is given by the (periodic) temporal extent of the lattice. $\varphi$ is the angle that parameterizes the imaginary chemical potential $\mu=i \varphi / \beta$. The individual canonical determinants $\operatorname{det}[D]^{(q)}$ appear as coefficients in the fugacity expansion of the grand canonical determinant $\operatorname{det}[D(\mu)]$,

$$
\operatorname{det}[D(\mu)]=\sum_{q} e^{\mu q \beta} \operatorname{det}[D]^{(q)} .
$$

Thus the representation with the canonical determinants is equivalent to the grand canonical formulation. In recent years several numerical simulations in the canonical formalism may be found in the literature [1] - [4], and were reviewed at this conference [5].

Canonical determinants do not only provide an alternative approach to lattice simulations with finite density, but also have interesting physical properties [6]. The grand canonical determinant $\operatorname{det}[D(\mu)]$ is a gauge invariant object and thus is a sum of products of closed loops which are dressed with link variables. The chemical potential is equivalent to a temporal fermionic boundary condition $\exp (i \varphi)$. This phase at the boundary is seen by the loops that wind around the compact time direction according to their total winding number and gives rise to a phase factor $\exp (i \varphi k)$ for a loop that winds $k$-times. The Fourier integral (1.1) over this boundary condition projects the grand canonical determinant to only those loops which have a net winding number of $k=q$ (see, e.g., [7] for a more detailed discussion of these relations). The fugacity expansion (1.2) thus may also be viewed as an expansion in terms of winding numbers of loops.

In a similar way all gauge invariant objects may be decomposed into sectors of loops with fixed winding number. This has been discussed for the chiral condensate, where it is hoped that the "dual chiral condensate", defined as the sector of the chiral condensate with winding 1 , might help to understand a possible relation between chiral symmetry restoration and deconfinement [8] $-[10]$.

\section{Center symmetry}

The canonical determinants have simple transformation properties under center transformations, where all temporal gauge links $U_{4}\left(\vec{x}, t_{0}\right)$ at a fixed time argument $t_{0}$ are multiplied with an element $z$ of the center of the gauge group, i.e., $U_{4}\left(\vec{x}, t_{0}\right) \rightarrow z U_{4}\left(\vec{x}, t_{0}\right)$. For the case of gauge group $\mathrm{SU}(3)$ the $z$ are the phases $z=1, \exp ( \pm i 2 \pi / 3)$. The gauge action and the gauge measure are invariant under center transformations. Consequently the expectation functional $\langle. .\rangle_{G}$ for the evaluation of observables in pure gauge theory is invariant under center transformations - as long as the center symmetry is not broken spontaneously. However, such a spontaneous breaking of the center symmetry takes place at high temperatures [11]. 

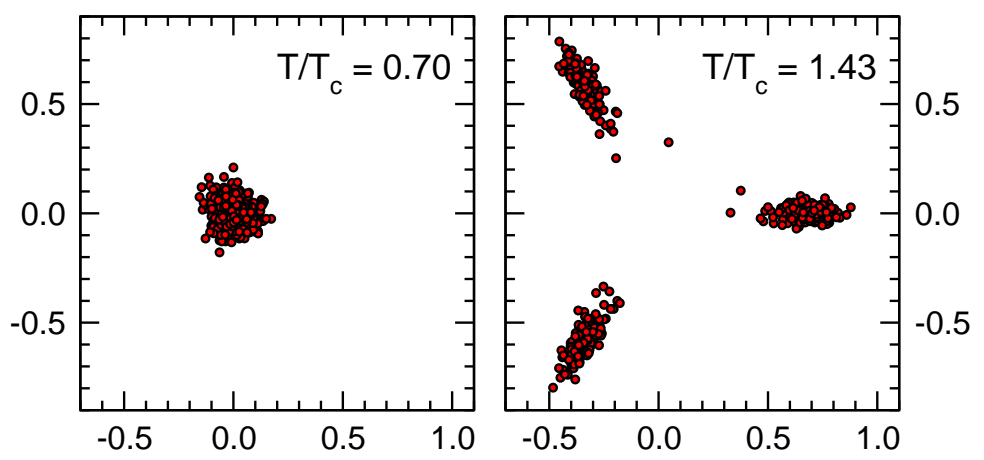

Figure 1: Scatter plots of Polyakov loop values in the complex plane for low (lhs.) and high temperature.

Observables may be classified with respect to their symmetry properties under center transformations. A simple example is the Polyakov loop $P$ which is the trace over a temporal gauge transporter that winds in a straight line once around compact time. As it winds once, it sees exactly one of the link variables $U_{4}\left(\vec{x}, t_{0}\right)$ which are transformed with the center element $z$ and we conclude that $P$ transforms as $P \rightarrow z P$. Since it transforms non-trivially, the Polyakov may be used as an order parameter for the breaking of center symmetry. Below the critical temperature $T_{c}$ its expectation value vanishes, while above $T_{c}$ this expectation value is finite.

The behavior of the Polyakov loop is illustrated in Fig. 1 where we show scatter plots of the Polyakov loop in the complex plane. The data are from 500 quenched gauge configurations on $8^{3} \times 4$ lattices generated with the Lüscher-Weisz gauge action [12]. We show two ensembles with temperatures of $T / T_{c}=0.7$ (lhs. plot) and $T / T_{c}=1.43$ (rhs.) according to the scale setting [13] with the Sommer parameter. Obviously for low temperatures the values of $P$ are compatible with zero, while they are non-vanishing above $T_{c}$, where the center symmetry is broken. We note that a true spontaneous breaking can happen only at infinite spatial volume. In that case the system will spontaneously select one of the three "islands" in the complex plane and only populate this one island.

Similar to the Polyakov loop we also may obtain the transformation properties of our canonical determinants $\operatorname{det}[D]^{(q)}$. We have already observed that they consist of loops with a net winding number of $q$ around compact time. Thus they have a net number of $q$ crossings of the time slice $t_{0}$ where the center transformation acts and consequently transform as

$$
\operatorname{det}[D]^{(q)} \longrightarrow z^{q} \operatorname{det}[D]^{(q)}=z^{q \bmod 3} \operatorname{det}[D]^{(q)},
$$

where in the last step we have used that $z=1, \exp ( \pm i 2 \pi / 3)$.

The canonical partition sums $Z^{(q)}$ for a fixed quark number $q$ are obtained as the expectation values of the canonical determinants using the pure gauge theory expectation functional $\langle. .\rangle_{G}$. As long as the center symmetry is unbroken, we find

$$
Z^{(q)}=\left\langle\operatorname{det}[D]^{(q)}\right\rangle_{G} \stackrel{\text { c.u. } !}{=} z^{q}\left\langle\operatorname{det}[D]^{(q)}\right\rangle_{G} \Longrightarrow Z^{(q)}=0 \text { for } q \bmod 3 \neq 0 .
$$

Thus in the low temperature phase, where the center symmetry is unbroken, the canonical partition sums $Z^{(q)}$ are non-vanishing only for quark sectors with vanishing triality, i.e., when $q$ is a multiple 

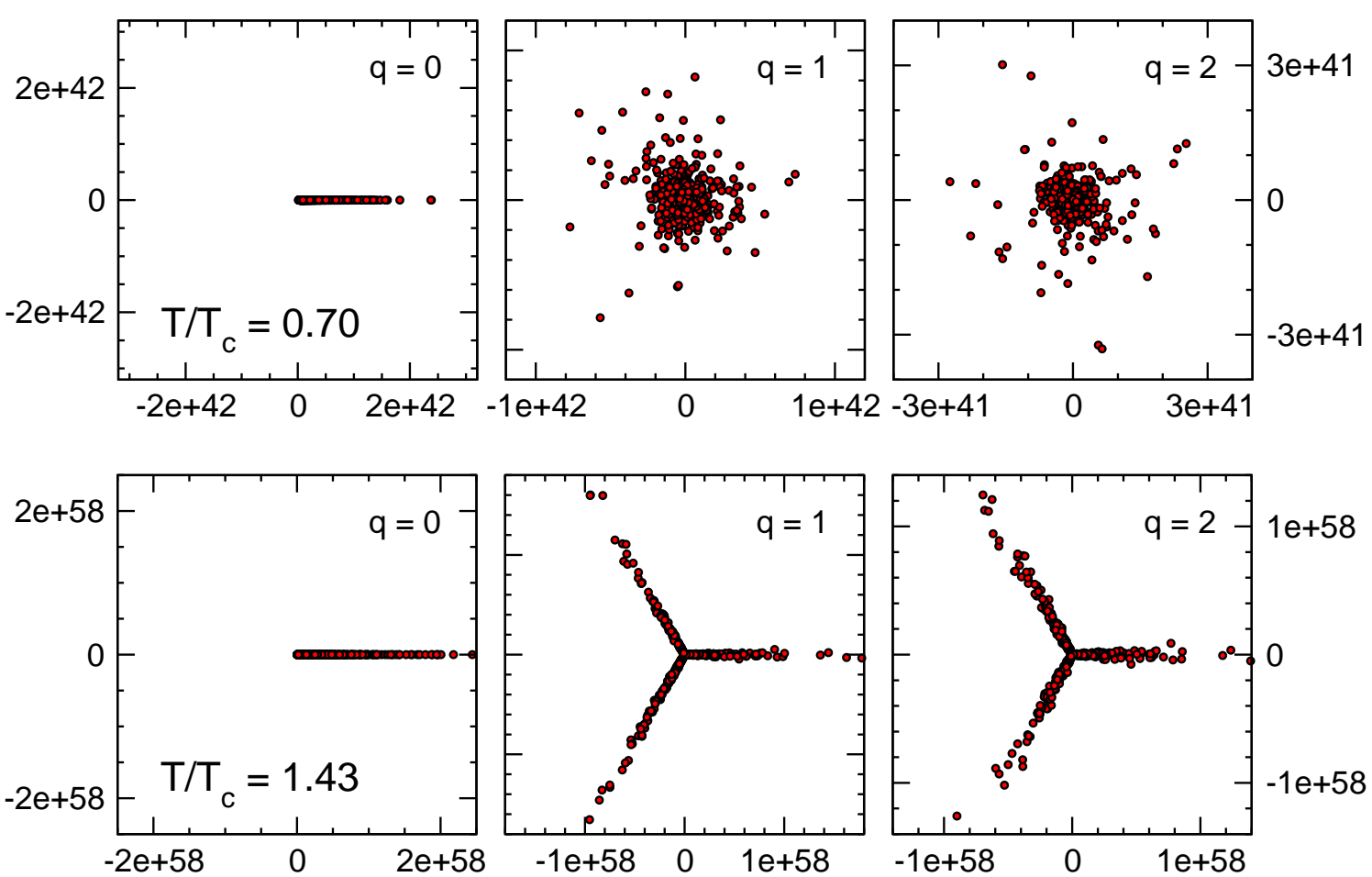

Figure 2: Scatter plots of the canonical determinants $\operatorname{det}[D]^{(q)}, q=0,1,2$ (left to right) in the complex plane. The top row is for $T / T_{c}=0.70$, the bottom for $T / T_{c}=1.43$.

of 3. The fact that the center symmetry is unbroken is crucial for the argument in (2.2), and we marked the step where we use that property by "c.u. !" for "center unbroken". We remark that the transformation properties (2.1) may be combined with the center properties of observables to derive selection rules for observables in the center symmetric phase [3], [7]. One finds that the total triality of an observable multiplied with a canonical determinant has to vanish for a non-vanishing contribution in the center symmetric phase.

The situation is different in the deconfined high temperature phase where center symmetry is broken spontaneously. The argument used in (2.2) may no longer be applied, and $Z^{(q)}$ can be non-vanishing also for $q \bmod 3 \neq 0$. In other words, above $T_{c}$ also canonical determinants $\operatorname{det}[D]^{(q)}$ with non-vanishing triality can have a non-vanishing expectation value.

In Fig. 2 we study the behavior of the canonical determinants $\operatorname{det}[D]^{(q)}$ below (top row of plots) and above $T_{c}$ (bottom) for quark numbers $q=0,1,2$ (plots from left to right). We show scatter plots of the values of the canonical determinants in the complex plane. The canonical determinants were evaluated as described in [7], using a dimensional reduction formula for determinants [14]. The lattice volume is $8^{3} \times 4$, the bare quark mass parameter in the fermion determinant is set to $m=$ $100 \mathrm{MeV}$, and the statistics is 500 configurations for $T<T_{c}$ (top row) and 800 configurations for $T>T_{c}$ (bottom).

For the zero triality case $q=0$ (left column of plots) the values of $\operatorname{det}[D]^{(0)}$ fall on the positive half of the real axis and thus give rise to a positive expectation value $Z^{(0)}$ both below and above $T_{c}$. For the non-vanishing triality sectors with $q=1,2$ (center and rhs. plots) the properties below 
and above $T_{c}$ are drastically different. Below $T_{c}$ the values of $\operatorname{det}[D]^{(q)}, q=1,2$, scatter around the origin in an isotropical distribution. Above $T_{c}$ the distribution is rather different and we observe the center symmetry pattern familiar from the Polyakov loop as shown in Fig. 1. We stress that for the canonical determinants the pattern is even much cleaner than for the Polyakov loop (Both, Fig. 1 and Fig. 2 were made with the same $8^{3} \times 4$ ensembles.).

Let us at this point address again the role of the infinite volume, which is necessary for a spontaneous breaking of the center symmetry. The high temperature data in Figs. 1 and 2 show the star-like pattern characteristic for the center broken phase. However, all three center sectors are populated equally and a naive averaging over all points in the scatter plots would give a vanishing expectation value for the Polyakov loop $P$, as well as the canonical determinants $\operatorname{det}[D]^{(q)}, q=1,2$ at all temperatures - a truly uninteresting outcome. To obtain the physically relevant result one must take into account that in the limit of infinite spatial volume, the system selects spontaneously only one of the three sectors, with the other two remaining empty. In a simulation on a finite lattice this may be taken into account by considering above $T_{c}$ the absolute values of symmetry breaking observables, i.e., $\langle|P|\rangle_{G}$ and $\left\langle\left|\operatorname{det}[D]^{(q)}\right|\right\rangle_{G}$.

\section{Distribution of the quark sectors}

Let us now study in more detail how the canonical determinants behave in the high temperature phase. In particular how sectors with different quark numbers behave relative to each other. As already discussed in the last section, above $T_{c}$, where the center symmetry is broken, also the sectors with $q$ mod $3 \neq 0$ can have non-vanishing expectation values. As also addressed there, one has to average the absolute value of the determinants, as long as one is on a finite volume where the center symmetry cannot be broken and all three center sectors are populated equally (if a proper Monte Carlo update is used).

In Fig. 3 we show the distribution $\left\langle\left|\operatorname{det}[D]^{(q)}\right|\right\rangle_{G} /\left\langle\operatorname{det}[D]^{(0)}\right\rangle_{G}$ as a function of $q$, i.e., we normalize with respect to the trivial sector with $q=0$. The data are for lattice size $8^{3} \times 4$, a quark mass of $m=100 \mathrm{MeV}$, and a statistics of 100 configurations. We show the results for different temperatures, ranging from $T=0.70 T_{c}$ to $T=1.43 T_{c}$

Below $T_{c}$ the distribution of the absolute value of the canonical determinants shows a Gaussian type of behavior with a rather narrow width. This width is increasing with temperature. Above $T_{c}$ the distribution remains Gaussian, but the width does not seem to grow any longer with temperature. We remark, however, at this point, that we work with a lattice size fixed in lattice units $\left(8^{3} \times 4\right)$, and change the temperature by varying the gauge coupling. Thus increasing the temperature also shrinks the spatial volume. This effect could mask a further widening of the distribution above $T_{c}$, but even if such widening persists, it is much smaller than the effect seen below $T_{c}$. A detailed finite volume analysis of the quark distribution must be left for future studies.

We conclude with discussing an important consistency check: Once the canonical determinants are known, one can try to sum up the fugacity expansion (1.2) and compare this sum to the grand canonical fermion determinant. The plots in Fig. 3 show that the canonical determinants quickly approach zero as $|q|$ increases, and a truncation of the fugacity expansion seems justified. We implemented such a test and summed the fugacity expansion, typically taking into account terms with $|q|$ up to values of 30-50. For moderate chemical potential of up to $a \mu \sim 0.1$ we found 

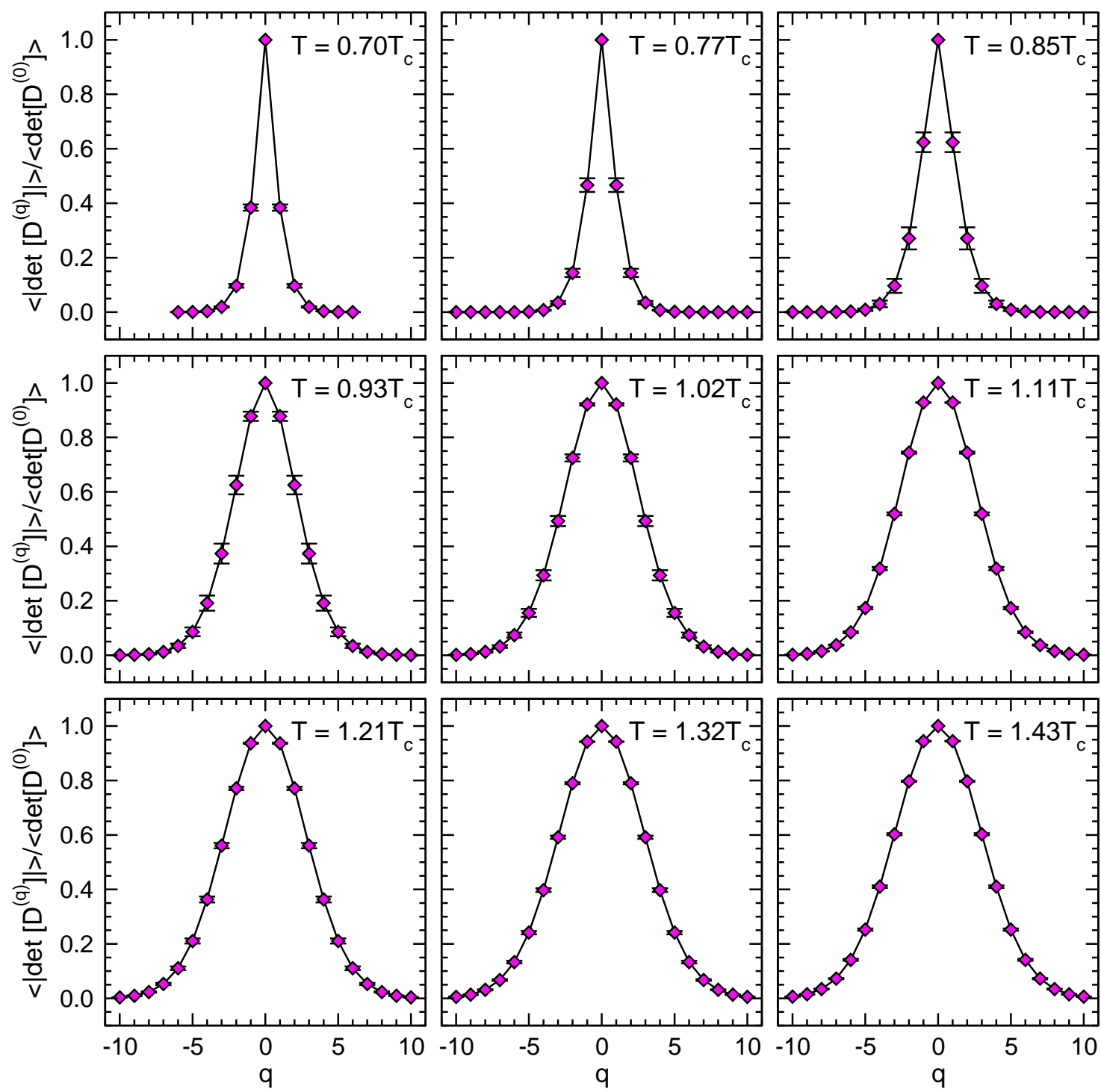

Figure 3: The distribution of $\left\langle\left|\operatorname{det}[D]^{(q)}\right|\right\rangle_{G} /\left\langle\operatorname{det}[D]^{(0)}\right\rangle_{G}$ as a function of the quark number $q$ for different values of the temperature.

excellent agreement between the fugacity sum and the grand canonical result, showing that the determination of the lowest canonical determinants is sufficiently accurate. For larger values of the chemical potential higher terms start to contribute which would have to be evaluated with higher accuracy.

\section{References}

[1] A. Li, X. Meng, A. Alexandru, K. F. Liu, PoS LATTICE2008, 178 (2008) [arXiv:0810.2349 [hep-lat]].

A. Li, A. Alexandru, K. F. Liu, PoS LAT2007, 203 (2007) [arXiv:0711.2692 [hep-lat]].

A. Alexandru, A. Li, K. F. Liu, PoS LAT2007, 167 (2007) [arXiv:0711.2678 [hep-lat]]. 
A. Alexandru, M. Faber, I. Horvath, K. F. Liu, Phys. Rev. D 72 (2005) 114513 [arXiv:hep-lat/0507020].

X. Meng, A. Li, A. Alexandru, K. F. Liu, PoS LATTICE2008, 032 (2008) [arXiv:0811.2112 [hep-lat]].

[2] P. de Forcrand, S. Kratochvila, Nucl. Phys. Proc. Suppl. 153 (2006) 62 [arXiv:hep-lat/0602024]; PoS LAT2005 (2006) 167 [arXiv:hep-lat/0509143]; Nucl. Phys. Proc. Suppl. 140 (2005) 514 [arXiv:hep-lat/0409072].

[3] S. Kratochvila, P. de Forcrand, Phys. Rev. D 73 (2006) 114512 [arXiv:hep-lat/0602005].

[4] S. Ejiri, Phys. Rev. D 78, 074507 (2008) [arXiv:0804.3227 [hep-lat]].

[5] A. Li, plenary talk at this conference.

[6] M. Faber, O. Borisenko and G. Zinovev, Nucl. Phys. B 444 (1995) 563 [arXiv:hep-ph/9504264].

[7] C. Gattringer, L. Liptak, arXiv:0906.1088 [hep-lat].

[8] C. Gattringer, Phys. Rev. Lett. 97 (2006) 032003 [arXiv:hep-lat/0605018].

F. Bruckmann, C. Gattringer, C. Hagen, Phys. Lett. B 647 (2007) 56 [arXiv:hep-lat/0612020].

E. Bilgici, F. Bruckmann, C. Gattringer, C. Hagen, Phys. Rev. D 77 (2008) 094007 [arXiv:0801.4051 [hep-lat]].

F. Bruckmann, C. Hagen, E. Bilgici, C. Gattringer, PoS CONFINEMENT8 (2008) 054 [arXiv:0812.2895 [hep-lat]].

E. Bilgici, et al, arXiv:0906.3957 [hep-lat].

[9] F. Synatschke, A. Wipf, C. Wozar, Phys. Rev. D 75 (2007) 114003 [arXiv:hep-lat/0703018].

F. Synatschke, A. Wipf, K. Langfeld, Phys. Rev. D 77 (2008) 114018 [arXiv:0803.0271 [hep-lat]].

[10] C. S. Fischer, Phys. Rev. Lett. 103 (2009) 052003 [arXiv:0904.2700 [hep-ph]].

C. S. Fischer, J. A. Mueller, arXiv:0908.0007 [hep-ph]; arXiv:0908.2530 [hep-ph].

J. Braun, L. M. Haas, F. Marhauser, J. M. Pawlowski, arXiv:0908.0008 [hep-ph].

K. Kashiwa, H. Kouno, M. Yahiro, arXiv:0908.1213 [hep-ph].

[11] L. McLerran, B. Svetitsky, Phys. Rev. D 24, 450 (1981).

[12] M. Lüscher, P. Weisz, Commun. Math. Phys. 97 (1985) 59 [Erratum-ibid. 98 (1985) 433].

G. Curci, P. Menotti, G. Paffuti, Phys. Lett. B 130 (1983) 205 [Erratum-ibid. B 135 (1984) 516].

[13] C. Gattringer, R. Hoffmann, S. Schaefer, Phys. Rev. D 65 (2002) 094503 [arXiv:hep-lat/0112024].

[14] J. Danzer, C. Gattringer, Phys. Rev. D 78 (2008) 114506 [arXiv:0809.2736 [hep-lat]]. P. Gibbs, Phys. Lett. B 172 (1986) 53. A. Borici, Prog. Theor. Phys. Suppl. 153 (2004) 335. 